\title{
Cloning and characterization of a UDP-glucose dehydrogenase gene from mulberry Broussonetia kazinoki $\times$ Broussonetia papyifera
}

\author{
R.H. JI ${ }^{1,2}$, Z. ZHANG ${ }^{3}$, X. GUO ${ }^{2}$, Y.L. BAO ${ }^{1}$, W.B. ZHANG*', X.F. LIN ${ }^{2}$, and S.L. BAI ${ }^{1 *}$ \\ College of Forestry, Inner Mongolia Agricultural University, Hohhot 010019, P.R. China ${ }^{1}$ \\ School of Life Sciences, Inner Mongolia University, Hohhot 010070, P.R. China ${ }^{2}$ \\ College of Biological Science, China Agriculture University, Beijing 100083, P.R. China ${ }^{3}$
}

\begin{abstract}
Uridine diphosphate glucose dehydrogenase (UGDH) is a key enzyme in the hemicellulose and pectin biosynthesis pathway and participates in the regulation of growth and development in plants. In this study, we isolated a $B p U G D H$ gene from paper mulberry (Broussonetia kazinoki $\times$ Broussonetia papyifera) and analyzed its function and expression characteristics. The results show that the $B p U G D H$ was expressed in all organs of paper mulberry with a higher expression in stems than in leaves and roots. A pBpUGDH::GUS gene construct was highly expressed in transgenic Arabidopsis thaliana seedlings, and its expression was induced by a low temperature, methyl jasmonate, gibberellin $\mathrm{A}_{3}$, ethylene, and auxin. The overexpression of $B p U G D H$ increased the soluble sugar content, promoted the accumulation of hemicellulose, and enhanced the vegetative growth of transgenic plants. These results provide a basis for regulating the growth and adaptability of paper mulberry and improving its utilization value via genetic modification of the $B p U G D H$ gene.
\end{abstract}

Additional key words: UGDH, hemicellulose, RACE, sugars, UDP-GlcA.

\section{Introduction}

The biosynthesis of hemicellulose and pectin in higher plants is regulated mainly by cytosolic UDP-glucose dehydrogenase (UGDH, EC1.1.1.22) (Labate et al. 2010) which uses $\mathrm{NAD}^{+}$as a coenzyme to catalyze the conversion of uridine diphosphate glucose (UDP-Glc) to UDP-glucuronate (UDP-GlcA), the key precursor in the synthesis of many nucleoside sugars including UDPgalacturonic acid, UDP-xylose, UDP-mannose, UDParabinose, and UDP-apiose (UDP-Api) (Campbell et al. 2000). These nucleoside sugars are further involved in the formation of polysaccharides in plants and participate in the formation of pectin and hemicellulose. Hemicellulose and pectin are key components of the cell wall and form a matrix that enhances the cell wall structure (Delmer et al. 1988, Witt 1992, Gibeaut and Carpita 1994, Gibeaut 2000). Up to $60 \%$ of the total cell wall polysaccharide content is derived directly or indirectly from UDP-GlcA
(Seitz et al. 2000).

A $U G D H$ gene was first isolated from the livers of Dutch pigs (Strominger et al. 1954), while the first higher plant $U G D H$ gene was cloned from soybean (Tenhaken and Thulke 1996). Subsequently, the $U G D H$ genes were isolated from the other species, such as Arabidopsis thaliana (Seitz et al. 2000), Escherichia coli (Campbell et al. 2000), Populus tremula $\times$ Populus tremuloides (Johansson et al. 2002), Dunaliella salina (He et al. 2005), Zea mays (Kärkönen et al. 2005), Boehmeria nivea (Liu et al. 2008), Eucalyptus grandis (Labate et al. 2010), Gossypium hirsutum (Pang et al. 2010), Hordeum vulgare (Matsumoto et al. 2011), Prunus persica (Sato et al. 2013), Ipomoea batatas (Lai et al. 2014), and Larix gmelinii (Li et al. 2017). The protein encoded by the UGDH gene has a molecular mass of approximately 40 - $53 \mathrm{kDa}$ (Robertson et al. 1996). UGDH proteins are generally considered to be localized in the cytoplasm and most are membranebound (Griffith et al. 2004, Rigg et al. 1998). Plant

Submitted 17 November 2019, last revision 24 June 2020, accepted 26 June 2020.

Abbreviations: CaMV - cauliflower mosaic virus; ETH - ethylene; $\mathrm{GA}_{3}$ - gibberellin $\mathrm{A}_{3}$; GUS - $\beta$-glucuronidase; IAA - indole acetic acid; MeJA - methyl jasmonate; MS - Murashige and Skoog; NAD - nicotinamide adenine dinucleotide; NPT II - neomycin phosphotransferase II gene; RACE - rapid amplification of cDNA ends; RT-PCR - reverse transcription polymerase chain reaction; UDP-Glc - uridine diphosphate glucose; UDP-GlcA - uridine diphosphate glucuronate; UGDH - uridine diphosphate glucose dehydrogenase; UGPase uridine diphosphate glucose pyrophosphorylase; WT - wild type.

Acknowledgments: We would like to thank Prof. Nakamura Kenzo in the Chubu University for kindly providing the pBI101-35::Gus$\mathrm{Hm}$ vector, and Prof. Qi Zhi in the Inner Mongolia University for kindly providing the pORE R1 vector. This study was supported by the National Natural Science Foundation of China (Nos. 31660213, 31560065, 31860206, and 31260168) and the Natural Science Foundation of Inner Mongolia (Nos. 2015MS0354 and 216MS0329).

Corresponding author: fax: (+86) 471 4301330; e-mail: baishulan2004@163.com 
UGDHs share high sequence homology with each other, and lower sequence homology with prokaryotic UGDHs. Plant UGDHs contain two functional domains, the NAD coenzyme binding site (G-A-G-Y-V-G-G) located at amino acids 8 - 14, and the catalytic site (G-F-G-G-S-C-FQ-K-D-I-L) located at amino acids 267 - 278. Other active residues, such as Pro89, Pro156, and Cys272 have been identified and are involved in catalytic reactions of the UGDH enzyme (Wang et al. 2013, Johansson et al. 2002).

In Prunus persica, the content of PpUGD1 (AB181204) mRNA is much higher in immature leaves than in mature leaves indicating a possible function in cell wall biosynthesis during leaf development. The change in the PpUGD1 mRNA content during peach fruit development corresponds to changes in the amount of cell wall material and the cell wall uronic acid content, and the expression of PpUGD1 is higher in the fruits of commercial peach cultivars than in native Japanese peach cultivars, suggesting a possible role of PpUGD1 in cell wall biosynthesis during peach fruit development (Sato et al. 2013). Expression of the P. tremula $\times$ P. tremuloides $U G D H$ gene (AF053973) was shown to be specific to xylem and young leaves and its transcription is strongly induced by sucrose, sorbitol, and PEG-8000, indicating it plays an important role in osmoregulation, while UGDH protein content is not affected, indicating regulation at the post-transcriptional or translational levels (Johansson et al. 2002). Expression of the DsUGDH gene (AY795899) of Dunaliella salina is rapidly induced by salt stress, indicating participation in the synthesis of polysaccharides and in salt tolerance in this algae (He et al. 2005). Five UGDHgenes (IbUDPGH1 KC509922, IbUDPGH2 KC509923, IbUDPGH3 KC509924, IbUDPGH4 KC509925, IbUDPGH5 KC509926) were identified in Ipomoea batatas which show different expression patterns; IbUDPGH1 is mainly expressed in the tuberous roots, IbUDPGH2 and IbUDPGH5 are expressed in stems, and the other $I b U G D H g e n e s$ are slightly expressed in different tissues (Lai et al. 2014). Four functional $U G D H$ genes (UGDH1 NM102419, UGDH2 AF424576, UGDH3 AY056200, UGDH4 BT006380) and one pseudogene were identified in the $A$. thaliana genome, and they exhibit distinct tissue-specific expression patterns during plant development (Klinghammer and Tenhaken 2007).

The UGDH plays a crucial role in sugar metabolism and plant cell wall formation. Klinghammer and Tenhaken (2007) showed that overexpression of the $A t U G D H$ gene in transgenic plants not only increases the polysaccharide content of the cell wall, but also produces specific phenotypes such as shortened aerial parts, a well-developed root system, increased secondary flowering, and purple seed epidermis. Heterologous expression of the L. gmelinii $U G D H$ gene (LC005487) in $A$. thaliana demonstrates that the $\operatorname{LgUGDH}$ gene enhances the vegetative growth, hemicellulose biosynthesis, and cold resistance of the transgenic plants (Li et al. 2017). Functional analysis of the $\mathrm{ZmUGDH}$ gene (ZmUGDH1 658123 and $\mathrm{ZmUGDH}$ 658125 in the GnpSeq database) from Z. mays using a mutation method shows that the content of matrix polysaccharide required for cell wall formation is decreased significantly in $\mathrm{ZmUGDH}$ mutant lines (Kärkönen et al. 2005). These results indicate that UGDH plays a key role in regulating growth and development in plants.

Paper mulberry is a multifunctional and common tree species that is widely used in the paper manufacturing, animal feed, and medicine industries, and plays an important role in environmental protection and ecological restoration (Li et al. 2011, Nagpal et al. 2011, Yan et al. 2011, Kuang et al. 2012). A previous research on paper mulberry focused mainly on developmental regulation and applications for breeding, animal feed, and medicine, with a limited amount of research devoted to its physiology, biochemistry, and molecular biology (Li et al. 2008, Sun et al. 2014). Therefore, further study on the molecular mechanism of the regulation of growth and development as well as stress tolerance in paper mulberry is necessary for rational utilization of this multifunctional and common tree species. Therefore, in this study, we isolated an ortholog of the $U G D H$ gene from paper mulberry and investigated features of its DNA sequence, its expression pattern, and its function in regulating growth, development, and coldtolerance. Our results may provide a basis for improving growth and adaptability of mulberry and increasing its utilization value through genetic modification of the BpUGDH gene.

\section{Materials and methods}

Plants: Paper mulberry [Broussonetia kazinoki Siebold $\times$ Broussonetia papyifera (L.) Went] cuttings were provided by the Lü He Agriculture and Forestry Limited Liability Company, Baotou, Inner Mongolia. The cuttings were planted in pots containing a $1: 1(\mathrm{~m} / \mathrm{m})$ mixture of soil and Vermiculite, and RNA and DNA were extracted from young leaves after 4 weeks of culture.

Seeds of Arabidopsis thaliana L. ecotype Columbia were sterilized with $75 \%(\mathrm{v} / \mathrm{v})$ ethanol and $2 \%(\mathrm{~m} / \mathrm{v})$ $\mathrm{NaClO}$ solution and then sown on 1/2 Murashige and Skoog (1962; MS) medium. After vernalization at a temperature of $4{ }^{\circ} \mathrm{C}$ for $3 \mathrm{~d}$, the seeds were cultured at $24{ }^{\circ} \mathrm{C}$, a $16-\mathrm{h}$ photoperiod, and an irradiance of $45 \mu \mathrm{mol} \mathrm{m} \mathrm{m}^{-2} \mathrm{~s}^{-1}$. Twoweek-old $A$. thaliana seedlings were transplanted into pots containing soil and grown under the same conditions as described above.

Isolation of $\boldsymbol{B p} \boldsymbol{U} \boldsymbol{G D H}$ cDNA: The total RNA was extracted from approximately $100 \mathrm{mg}$ of young paper mulberry leaves using the MiniBEST plant RNA extraction kit (Takara, Beijing, China). First-strand cDNA was synthesized from $1 \mu \mathrm{g}$ of total RNA using the TransScript first-strand cDNA synthesis SuperMix reverse transcription kit (TransGen Biotech, Beijing, China) at $42{ }^{\circ} \mathrm{C}$ for $30 \mathrm{~min}$ with $1 \mathrm{~mm}^{3}$ of $100 \mu \mathrm{M}$ oligodT adaptor primer. Degenerate PCR was performed using the degenerate primer pairs $\mathrm{F} 1$ and $\mathrm{R} 1$, and $\mathrm{F} 2$ and $\mathrm{R} 2$ (Table 1 Suppl.) designed by Li et al. (2017). The first PCR reaction was performed, using $2.5 \mathrm{~mm}^{3}$ of $10 \times$ TransStart buffer (TransGen Biotech), $2 \mathrm{~mm}^{3}$ of $2.5 \mathrm{mM}$ dNTPs, $0.2 \mathrm{~mm}^{3}$ of 2.5 units of TransStart Taq DNA polymerase 
(TransGen Biotech), $1 \mathrm{~mm}^{3}$ of $50 \mu \mathrm{M}$ primers (F1 and $\mathrm{R} 1$ ), and $1 \mathrm{~mm}^{3}$ of cDNA in a $25-\mathrm{mm}^{3}$ volume, with the following conditions: 35 cycles of $94{ }^{\circ} \mathrm{C}$ for $30 \mathrm{~s}, 48{ }^{\circ} \mathrm{C}$ for $60 \mathrm{~s}$, and $72{ }^{\circ} \mathrm{C}$ for $1 \mathrm{~min}$, followed by $72^{\circ} \mathrm{C}$ for $5 \mathrm{~min}$ as a final extension. The second PCR was performed with primers (F2 and R2) under the same conditions as the first PCR. First strand cDNAs for 5'- and 3'-rapid amplification of cDNA ends (RACE) were synthesized using $1 \mu \mathrm{g}$ of the total RNA with a SMARTer RACE 5'/3' kit (Takara) according to the manufacturer's instructions. The $5^{\prime}$ - and 3'-RACE fragments of the $B p U G D H$ gene were amplified by $5^{\prime} / 3^{\prime}-$ RACE PCR using the gene-specific primers 5 'R1 and 5'R2/3'F1 and 3'F2 (Table 1 Suppl.) and the Universal Primer Mix (UPM) and Universal Primer Mix short (UPM short) provided by a SMARTer RACE kit under the following conditions: 25 cycles of $94^{\circ} \mathrm{C}$ for $30 \mathrm{~s}, 68^{\circ} \mathrm{C}$ for $30 \mathrm{~s}$, and $72^{\circ} \mathrm{C}$ for $2 \mathrm{~min}$, followed by $72^{\circ} \mathrm{C}$ for $5 \mathrm{~min}$ as a final extension.

A full-length $B p U G D H$ cDNA fragment containing the complete coding region was obtained using standard PCR with the BpUGDH-full-F and BpUGDH-full-R primers (Table 1 Suppl.) under the following conditions: 35 cycles of $94{ }^{\circ} \mathrm{C}$ for $30 \mathrm{~s}, 59{ }^{\circ} \mathrm{C}$ for $30 \mathrm{~s}$, and $72{ }^{\circ} \mathrm{C}$ for $2 \mathrm{~min}$, followed by $72{ }^{\circ} \mathrm{C}$ for $5 \mathrm{~min}$ as a final extension. The products of the above PCRs were inserted into the pMD-19T vector and transformed into Trans1-T1 Phage Resistant competent cells (TransGen Biotech, Beijing, China), and DNA sequencing was performed by the Beijing Genomics Institute (Beijing, China).

Characterization of the $B p U G D H$ gene: Analysis of $B p U G D H$ expression was performed with real-time PCR using the $B p U G D H$-specific primers BpUGDHSP-F and BpUGDH-SP-R (Table 1 Suppl.). BpActin (ID in transcriptomic data: T7-27886, one of the unigenes annotated as BpActin was used for the primer design, in the NCBI Short Read Archive (SRA) database under accession number SRP029966) expression was assessed using BpActin-F and BpActin-R primers (Li et al. 2007, Peng et al. 2015) and served as an internal control. Firststrand cDNA was synthesized from total RNA $(2 \mu \mathrm{g})$ prepared from leaves, stems, and roots of paper mulberry seedlings using TransScript All-in-One first-strand cDNA synthesis SuperMix for qPCR (one-step gDNA removal) (Takara). The cDNA was diluted ten-fold and used as template for RT-PCR analysis with a LightCycler 480 real time PCR platform (Roche, San Francisco, CA, USA). All experiments were repeated three times, and the data were analyzed with the $S A S$ software by the comparative CT $\left(2^{-\Delta \Delta \mathrm{Ct}}\right)$ method as previously described (Livak and Schmittgen 2001).

Cloning and sequence analysis of the $B p U G D H$ promoter: The $B p U G D H$ promoter sequence was isolated using a Genome Walking kit (Takara) with the BpUGDHspecific primers BpUGDH-P-SP1, BpUGDH-P-SP2, and BpUGDH-P-SP3 (Table 1 Suppl.) and the arbitrary degenerate primers $\mathrm{AD} 1, \mathrm{AD} 2$, and $\mathrm{AD} 3$ according to the manufacturer's instructions. Sequence analysis of the $B p U G D H$ promoter was carried out using the Plant CARE database (Lescot et al. 2002) and Berkeley Drosophila genome project $(B D G P)$.

Construction of pBI101-BpUGDH and pORE R1pUGDH::GUS binary vectors: The binary expression vectors, pBI101-BpUGDH and pORE R1-pUGDH::GUS were constructed by homologous recombination and used for functional analysis and expression detection of $B p U G D H$ in transformed $A$. thaliana plants. Primers for amplifying the $B p U G D H$ open reading frame (BpUGDHBam $\mathrm{H} 1-\mathrm{F}$ and BpUGDH-BamH1-R) and for amplifying the $B p U G D H$ promoter sequence (BpUGDH-Xbal-F and BpUGDH-Xbal-R) (Table 1 Suppl.) were designed to overlap sequences of the pBI101-Km-35S:: Gus-Hm vector (provided by Professor Nakamura Kenzo; Chubu University, Japan) or the pORE R1 vector (provided by Professor Qi Zhi; Inner Mongolia University, China), respectively. The target fragments were amplified using the TransStart FastPfu DNA polymerase kit (TransGen Biotech) in PCR reactions containing $10 \mathrm{~mm}^{3}$ of $5 \times$ TransStart FastPfu buffer, $4 \mathrm{~mm}^{3}$ of $2.5 \mathrm{mM}$ dNTPs, $1 \mathrm{~mm}^{3}$ of 2.5 units of TransStart FastPfu DNA polymerase (TransGen Biotech), $1 \mathrm{~mm}^{3}$ of $10 \mu \mathrm{M}$ primers, and $2 \mathrm{~mm}^{3}$ of cDNA in a $50-\mathrm{mm}^{3}$ volume with the following conditions: 35 cycles of $95{ }^{\circ} \mathrm{C}$ for $20 \mathrm{~s}, 68^{\circ} \mathrm{C}$ for $20 \mathrm{~s}$, and $72{ }^{\circ} \mathrm{C}$ for $2 \mathrm{~min}$, followed by $72{ }^{\circ} \mathrm{C}$ for $5 \mathrm{~min}$ as a final extension. Homologous recombination of linearized vector (pBI101-Km-35S::Gus-Hm digested with BamHI and pORE R1 digested with $X b a \mathrm{I}$ ) and target fragment was performed using the $p E A S Y$-Uni seamless cloning and assembly kit (TransGen Biotech). The reaction contained $5 \mathrm{~mm}^{3}$ of $2 \times$ assembly mix, 0.02 pmole of linearized vector, and 0.04 pmole of target fragments in a $10-\mathrm{mm}^{3}$ volume at $50{ }^{\circ} \mathrm{C}$ for $15 \mathrm{~min}$.

Screening and identification of transgenic $A$. thaliana lines: Recombinant plasmids were introduced into the Agrobacterium tumefaciens strain GV3101 and used to transform $A$. thaliana via the simplified in-plant infiltration method described by Kim et al. (1999). Transgenic lines were obtained by screening on $1 / 2 \mathrm{MS}$ medium containing kanamycin (40 $\left.\mathrm{mg} \mathrm{dm}^{-3}\right)$ and confirmed by PCR analysis of genomic DNA from wild-type and transgenic A. thaliana. Genomic DNA PCR was performed using the $B p U G D H$ promoter/gene-specific primers with annealing at $55^{\circ} \mathrm{C}$ for the $\mathrm{BpUGDH}$ promoter and $59{ }^{\circ} \mathrm{C}$ for the $B p U G D H$ gene for $30 \mathrm{~s}$. RT-PCR was performed to detect transgene expression with the vector-specific primers Gus-F and Gus-R (Table 1 Suppl.), and BpUGDH genespecific primers BpUGDH-full-F and BpUGDH-full-R (Table 1 Suppl.). AtActin2 gene (AT3G18780 AK317453) expression (Atactin2-F and Atactin2-R primers, Lin et al. 2008) served as an internal control. PCR was performed using the following conditions: $94{ }^{\circ} \mathrm{C}$ for $3 \mathrm{~min}$; 35 cycles of $94{ }^{\circ} \mathrm{C}$ for $30 \mathrm{~s}, 65{ }^{\circ} \mathrm{C}($ gusA $), 59{ }^{\circ} \mathrm{C}(B p U G D H)$, or $55^{\circ} \mathrm{C}$ (AtActin) for $30 \mathrm{~s}$, and $72{ }^{\circ} \mathrm{C}$ for $1 \mathrm{~min}$, followed by $72{ }^{\circ} \mathrm{C}$ for $5 \mathrm{~min}$ as a final extension.

$\boldsymbol{\beta}$-Glucuronidase staining: $\beta$-Glucuronidase (GUS) expression driven by the $B p U G D H$ promoter in 
A. thaliana plants transformed with the pORE R1pUGDH::GUS construct was detected using a GUS staining kit (HuaYueyang, Beijing, China) at various plant growth stages. The plant tissues were immersed in GUS staining solution at $37^{\circ} \mathrm{C}$ overnight, decolorized 2 3 times with $70 \%(\mathrm{v} / \mathrm{v})$ ethanol to remove chlorophyll, and observed under a Nikon SMZ1000 (Tokyo, Japan) microscope. To investigate the effects of phytohormones on GUS expression at various growth stages of transgenic A. thaliana, seedlings transplanted onto $1 / 2 \mathrm{MS}$ medium supplemented with $100 \mu \mathrm{M}$ methyl jasmonate (MeJA), $100 \mu \mathrm{M}$ ethylene (ETH), $50 \mu \mathrm{M}$ indole acetic acid (IAA), or $50 \mathrm{mg} \mathrm{dm}^{-3}$ gibberellin $\mathrm{A}_{3}\left(\mathrm{GA}_{3}\right)$ and 6,13 , and 20 -d-old plants were used for GUS staining. To investigate GUS expression in flowers and seeds, transgenic plants grown in pots were sprayed with $100 \mu \mathrm{M}$ MeJA, $100 \mu \mathrm{M}$ ETH, $50 \mu \mathrm{M}$ IAA, or $50 \mathrm{mg} \mathrm{dm}^{-3} \mathrm{GA}_{3}$ at 12 -h intervals and GUS staining was performed after $48 \mathrm{~h}$. To investigate the effect of low temperature on GUS expression, 4, 11, 18, 25, and 32-d-old transgenic $A$. thaliana plants were transferred to a $4{ }^{\circ} \mathrm{C}$ chamber and GUS staining was performed after $48 \mathrm{~h}$.

Phenotypic analysis of transgenic A. thaliana: Individual kanamycin-resistant plants were self-pollinated to establish lines homozygous for the transgene. All analyses were carried out with homozygous lines. Physical characteristics including length and maximum width of the fifth rosette leaf, number of cauline branches, internode distances, and plant height were measured in 40-d-old wild-type and transgenic $A$. thaliana plants, while root lengths were measured in 28-d-old plants.

Cold tolerance of transgenic $A$. thaliana: To investigate the cold tolerance of transgenic $A$. thaliana, 8-d-old wildtype and transgenic plants were transferred to $4{ }^{\circ} \mathrm{C}$, and their fresh masses were recorded after $0,3,7$, and $14 \mathrm{~d}$.

Activity of UGDH: Protein was extracted from the fifth rosette leaves of 30-d-old wild-type and transgenic A. thaliana plants as previously described ( $\mathrm{Li}$ et al. 2017) and UGDH activity was measured using a UGDH activity detection kit (COMIN, Suzhou, China). As UGDH catalyzes the reaction of D-glucose and $\mathrm{NAD}^{+}$to generate UDP-GlcA and NADH, UGDH activity was determined by measuring the change in absorbance at $340 \mathrm{~nm}$ caused by reduction of $\mathrm{NAD}^{+}$to $\mathrm{NADH}$ using a Spectramax I303030923 microplate reader (MD, San Jose, CA, USA).

Determination of sugar, lignin, cellulose, and hemicellulose content: The sugar content of the fifth rosette leaves of 30-d-old $A$. thaliana plants were measured using specific detection kits (COMIN, Suzhou, China) according to the manufacturer's instructions. Absorbance values at 505, 480, 620, 480, and $620 \mathrm{~nm}$ were measured using a Spectramax 1303030923 microplate reader, and the content of glucose, fructose, soluble sugars, sucrose, and starch were calculated. Lignin, cellulose, hemicelluloses, and pectin content in stems of 45-d-old $A$. thaliana plants were measured by detecting absorbance values at 620,540 , 530 , and $280 \mathrm{~nm}$ using specific detection kits (COMIN) according to the manufacturer's instructions.

Data analyses: All data are expressed as means \pm standard deviations (SDs). Statistical differences were assessed by one-way analysis of variance (ANOVA) using Fisher's least significant difference (LSD) test. The significance of differences between means was identified at a probability value of 0.05 . All statistical analyses were performed using the Sigma Plot v12.0 software (Systat Software, San Jose, CA, USA).

\section{Results}

Nested RT-PCR using degenerate primer sets was used to isolate a 752-bp $B p U G D H$ fragment. Primers for RACE were then generated and 3 ' and 5' RACE were performed to isolate a full-length $B p U G D H$ cDNA sequence of $1658 \mathrm{bp}$ (DDBJ, accession No. LC457701), which encoded a protein of 480 amino acids with a theoretical molecular mass of $53.1 \mathrm{kDa}$ and a $\mathrm{pI}$ of 5.98. BLAST $X$ analysis showed that the cloned $B p U G D H$ sequence shared high sequence similarity with homologous genes from Vitis vinifera (VvUGDH XP_010661131.1), Morus notabilis (MnUGDH XP_010089612.1), Ziziphus jujuba (ZjUGDH XP_015879845.1), B. nivea (BnUGDH ABM55267.3), Citrus sinensis (CsUGDH XP 006482729.1), Juglans regia (JrUGDH XP_018816674.1̄), A. thaliana (AtUGDH BAB02581.1), and Medicago truncatula (MtUGDH XP_003621403.1)(Fig.1 Suppl.). The encoded BpUGDH protein contained all the conserved features of plant UGDHs including the NAD coenzyme binding site (G-A-G-Y-V-G-G) located at amino acids 8 - 14, the catalytic site (G-F-G-G-S-C-F-Q-K-D-I-L) located at amino acids 267 - 278, and other active residues, such as Pro residues at positions 89 and 156, which were conserved in all of the eukaryotic UGDHs and are believed to correspond to the main bends in the protein structure (Wang et al. 2013), two Lys residues at positions 217 and 330 which correspond to Lys 216 and Lys 335 of $P$. tremula $\times P$. tremuloides UGDH (PtUGDH AAF04455.1) (one of these Lys residues is probably involved in the conversion of UDP-glucose to UDP-aldehydoglucose), and an important Cys residue at position 272 which is involved in the UGDH-catalyzed conversion of UDPaldehydoglucose to UDP-GlcA (Wang et al. 2013) (Fig. 1 Suppl.).

We evaluated tissue-specific expression of $B p U G D H$ by real time RT-PCR analysis of RNA extracted from roots, stems, and leaves of 4-week-old paper mulberry seedlings. The results showed that the $B p U G D H$ gene was expressed in all organs, with higher expression in stems than in leaves and roots (Fig. 1).

We isolated the 1324-bp BpUGDH promoter sequence using a genome walking method, and analyzed its DNA sequence using the online software packages PLACE (http://bioinformatics.psb. ugent.be/webtools/plantcare/ $\mathrm{html} /$ ) and BDGP (http://www.fruitfly.org/seq_tools/ promoter.html). The transcription initiation site was identified as an adenosine (A) located $18 \mathrm{bp}$ upstream of 
the start codon (Fig. 2 Suppl.), and functional cis-elements were identified including not only the CAAT box and the TATA box common in eukaryotic genes, but also response elements to phytohormones such as MeJA, $\mathrm{GA}_{3}$, IAA, and $\mathrm{ETH}$, suggesting that $\mathrm{Bp} U G D H$ expression is regulated by phytohormones. In addition, elements responsive to abiotic stress, such as low temperature, were also found in the $B p U G D H$ promoter region (Table 2 Suppl.). These results suggest that $B p U G D H$ is involved in the regulation of growth and development, as well as in the response to abiotic stress, including cold.

To further understand $B p U G D H$ expression characteristics, we introduced a pORE-R1pBpUGDH::GUS expression vector into $A$. thaliana and confirmed $B p U G D H$ promoter-driven GUS expression by RT-PCR analysis. We investigated the pattern of $B p U G D H$ expression in transgenic $A$. thaliana plants at various growth stages (6-, 13-, 20-, 27-, and 34-d-old plants) by GUS histochemical staining (Fig. 2). GUS was expressed in all organs of the transgenic plants and its expression was induced by low temperature, MeJA, GA $\mathrm{GA}_{3}$ IAA, and ETH (Fig. 2A-F). Higher GUS expression was detected in young seedling, including leaves, stems, and roots, while its expression decreased in mature organs (Fig. $2 A-C$ ), corresponding to the role of UGDH in cell wall biosynthesis. After flowering, higher GUS expression was detected in reproductive organs (Fig. $2 D-E$ ), including petals, calyx, stamens, and siliques, while no GUS staining was observed in seeds (Fig. $2 F$ ).

To further analyze the features and functions of $B p U G D H$, we constructed the pBI101-BpUGDH plasmid containing the $B p U G D H$ cDNA sequence with expression driven by the CaMV35S promoter (Fig. $3 A$ ) and introduced it into A. thaliana. Expression of the $B p U G D H$ transgene was confirmed by RT-PCR analysis in four homozygous transgenic lines produced by self-pollination (Fig. 3B). UGDH activity was measured in the four transgenic lines using the fifth rosette leaf of 30-d-old plants to confirm $B p U G D H$ expression at the translation level. UGDH activity was higher in the four $B p U G D H$ transgenic lines than in the wild-type plants (Fig. 3C).

Overexpression of $B p U G D H$ in transgenic A. thaliana resulted in distinct phenotypes at various developmental

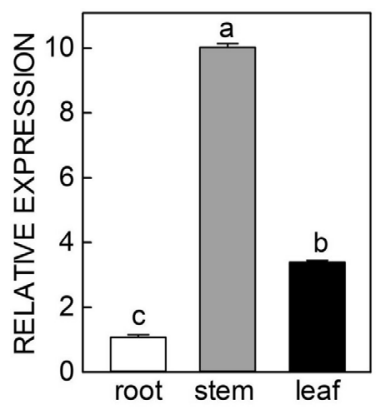

Fig. 1. Relative Broussonetia papyifera uridine diphosphate glucose dehydrogenase expressions in roots, stems, and leaves of paper mulberry determined by real time RT-PCR analysis. Error bars represent SDs of three independent biological replicates. Different lowercase letters represent significant differences between each group of samples $(P<0.05)$. stages, compared to the wild-type plants. There was no significant difference in the growth rate of the aboveground parts between wild-type and transgenic plants within two weeks after germination, while 28-d-old transgenic seedlings exhibited longer and more of welldeveloped roots (Fig. 4A). After transplanting into soil, the transgenic plants grew faster than the wild-type plants (Fig. 4B,C). To precisely analyze phenotypic differences between the wild-type and transgenic plants, we measured morphological characteristics of 40-d-old plants including the length and width of the fifth rosette leaves, the number of cauline branches, internode distances, and plant height. All of the transgenic plants were taller than the wild-type plants, and significant differences in leaf width, internode lenght, and root length were observed between wild-type and $B p U G D H$ transgenic plants (Fig. $4 A, D$ ). These results demonstrated that overexpression of $B p U G D H$ enhanced vegetative growth in the transgenic plants. The content of sucrose, fructose, and soluble sugars of the four $B p U G D H$ transgenic lines increased significantly relative to the wild-type plants, whereas the glucose content decreased significantly and no significant difference in starch content was observed (Fig. 5). The hemicellulose content in the stems of the four transgenic lines was significantly higher than in the wild-type plants, the cellulose content was significantly lower than in wild-type and no significant difference in lignin and pectin content was observed (Fig. 6). These results demonstrated that the $B p U G D H$ gene plays a role in controlling polysaccharide metabolism and biosynthesis of secondary cell walls.

To investigate the cold-tolerance of transgenic A. thaliana plants, 8 -d-old seedlings were cultured at $4{ }^{\circ} \mathrm{C}$ for $0,3,7$, and $14 \mathrm{~d}$, and fresh masses were measured (Fig. 7). There was no significant difference in fresh mass between the wild type and the four transgenic lines during 0 - $3 \mathrm{~d}$ of culture at $4{ }^{\circ} \mathrm{C}$, while damage induced by low temperature began to appear in wild-type plants after $7 \mathrm{~d}$, and the fresh masses of wild-type plants were significantly lower than those of the four transgenic lines. These results showed that overexpression of $B p U G D H$ enhanced coldtolerance in transgenic $A$. thaliana plants. To examine expression of $B p U G D H$ under the cold stress conditions, 4-week-old paper mulberry tissue culture seedlings were grown at $4{ }^{\circ} \mathrm{C}$ for $0,6,12,24,48,72 \mathrm{~h}$, respectively, and the expressions of $B p U G D H$ were measured. Real-time PCR results show that expression of $B p U G D H$ increased to a maximum at $6 \mathrm{~h}$ after culture under $4{ }^{\circ} \mathrm{C}$ and with the prolongation of stress time, the relative expression decreased slowly, and the expression was lowest at $72 \mathrm{~h}$ after culture at $4{ }^{\circ} \mathrm{C}$ (Fig. 3 Suppl.). These results indicate that the $B p U G D H$ gene could play an important role in adaptation of paper mulberry plants to low temperature.

\section{Discussion}

Polysaccharides essential for plant cell wall biosynthesis include cellulose, hemicelluloses, and tannins. The UGPase is an important regulatory enzyme in the biosynthesis of cellulose and callose and catalyzes the 
R.H. JI et al.

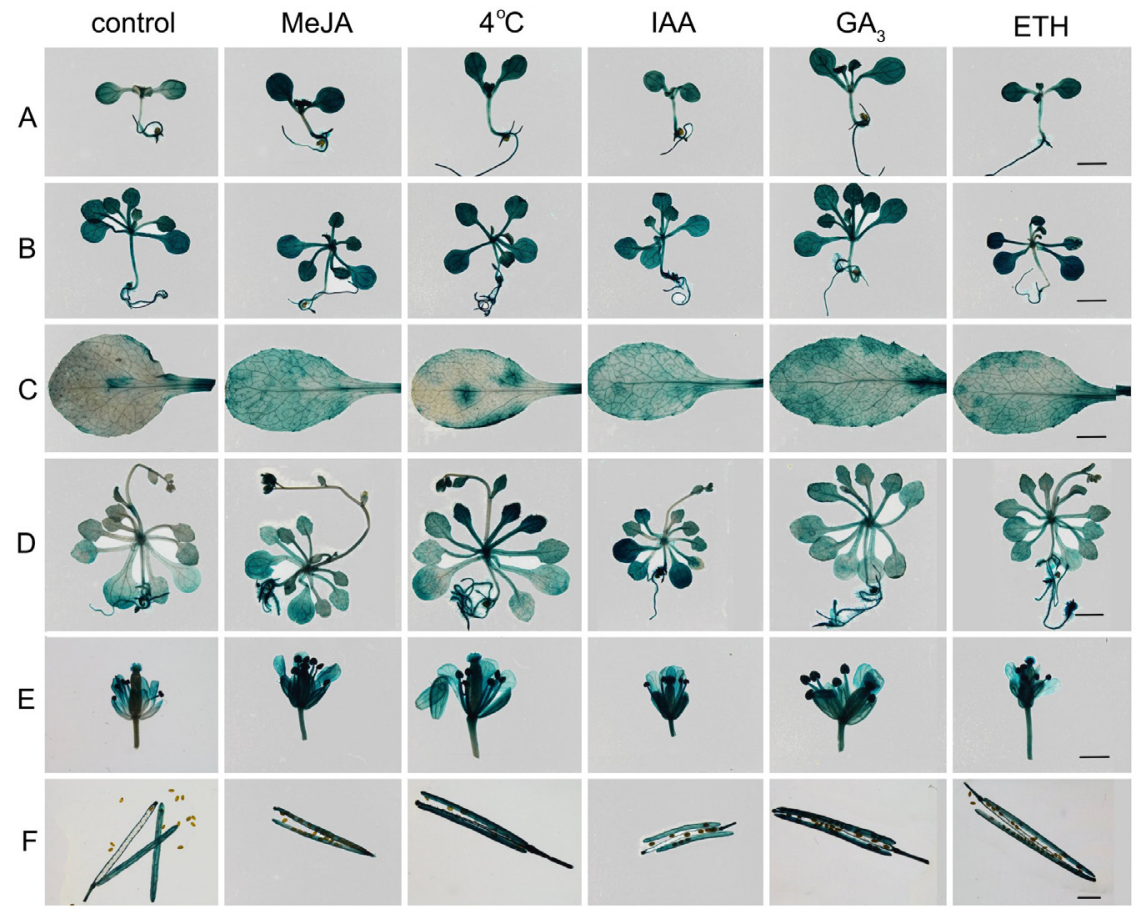

Fig. 2. $\beta$-Glucuronidase staining transgenic Arabidopsis thaliana tissues at various growth stages under different induction conditions; $A$ - 6-d-old seedlings, $B$ - 13-d-old plants, $C$ - leaves of 20-d-old plants, $D$ - 20-d-old plants, $E$ - flowers of 27-d-old plants, $F$ - pods of 34-d-old plants. MeJA - methyl jasmonate, $4{ }^{\circ} \mathrm{C}$ - cold treatment, IAA - indole acetic acid, $\mathrm{GA}_{3}$ - gibberellin $\mathrm{A}_{3}$, ETH - ethylene. Scale bars $-2 \mathrm{~mm}$.
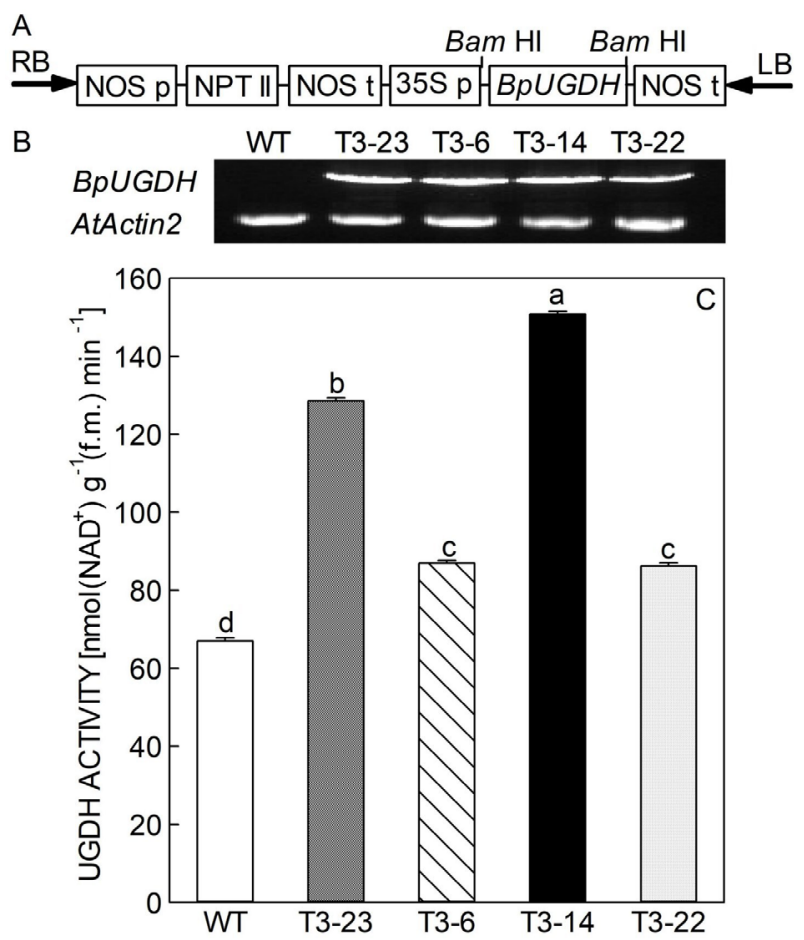

Fig. 3. Molecular identification of transgenic Arabidopsis thaliana lines. A - diagram of the T-DNA region of the pBI101-BpUGDH vector used to produce transgenic Broussonetia papyifera uridine diphosphate glucose dehydrogenase (BpUGDH) plants (35Sp cauliflower mosaic virus $35 \mathrm{~S}$ promoter, NOS p - nopaline synthase promoter, NOS t - nopaline synthase terminator, NPT II - neomycin phosphotransferase II gene, RB - right border, LB - left border). $B$ - confirmation of $B p U G D H$ transgene expression in various transgenic lines by RT-PCR analysis; the AtActin2 gene served as an internal control. $C$ - uridine diphosphate glucose dehydrogenase (UGDH) activities in wild-type and transgenic lines. Error bars represent SDs of three independent biological replicates. Different letters indicate significant differences $(P<0.05)$. 
reversible conversion of glucose-1-phosphate and uridine triphosphate to UDP-Glc and pyrophosphate (Winter and Huber 2000, Kleczkowski et al. 2004, Li et al. 2014). UDP-Glc is converted by UGDH to UDP-GlcA, which is involved in the biosynthesis of hemicellulose and pectin. As a key enzyme in the hemicellulose biosynthesis pathway, UGDH promotes vegetative growth of plants by regulating the metabolism of polysaccharides in plants to promote hemicellulose deposition and cell wall formation (Li et al. 2017). Therefore, it is important to analyze the expression characteristics and roles of UGDH in regulating the growth and development of plants.

In this study, we cloned a $U G D H$ ortholog from paper mulberry. It shares high sequence similarity with
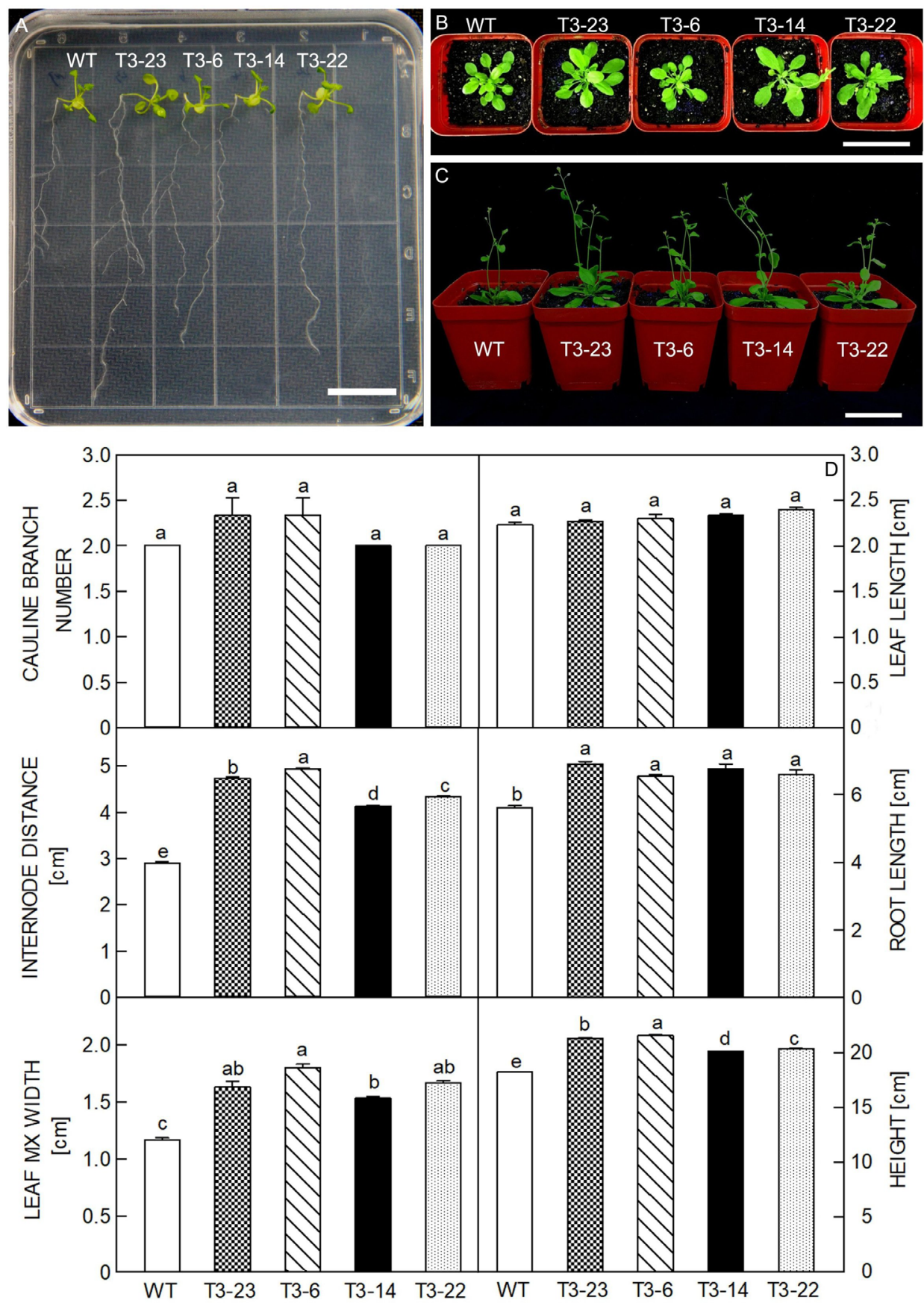

Fig. 4. Effects of Broussonetia papyifera uridine diphosphate glucose dehydrogenase overexpression on transgenic Arabidopsis thaliana plants. $A$ - 28-d-old wild-type and transgenic plants (T3-23, T3-6, T3-14, and T3-22 lines) cultivated on vertical plates showing differences in root growth and development (scale bar - $1.6 \mathrm{~cm}$ ). $B-20$-d-old transgenic plants showing a better growth than wild-type plants (left) (scale bar - $5 \mathrm{~cm}) . C$ - 35-d-old transgenic plants considerably taller than wild-type plants (left) $(s c a l e$ bar $-5 \mathrm{~cm}) . D-$ morphological analysis of 40-d-old wild-type and transgenic plants including the length and width of the fifth rosette leaves, the number of cauline branches, internode distances, and plant height. Error bars represent SDs of three independent biological replicates with six plants. Different letters indicate significant differences $(P<0.05)$. 
the $U G D H$ genes of other plant species and contains all the conserved features of plant UGDH proteins (Fig. 1 Suppl.), indicating its function in converting UDP-Glc to UDP-GlcA. Real time RT-PCR analysis showed that the $B p U G D H$ gene was expressed in all plant organs with higher expression in stems than in leaves and roots (Fig. 1), which is similar to the expression of the $L g U G D H$ gene from Larix gmelinii ( $\mathrm{Li}$ et al. 2017) and the BnUGDH gene (EF178294) from Boehmeria nivea (Liu et al. 2008). $L g U G D H$ showed the highest expression in stems, followed by leaves and roots. The $B n U G D H$ gene was expressed in roots, stems, leaves, and phloem, with the highest expression in stems (Liu et al. 2008), while the $U G D H$ gene in $G$. $\max (G m U G D H$ U53418.1) was highly expressed in radicle roots (Tenhaken and Thulke 1996).
Previous studies have shown that $U G D H$ expression is more active in young and rapidly developing tissues (Stewart 1998). In this study, GUS staining showed, that during expression driven by the $B p U G D H$ promoter, GUS was strongly expressed in young and developing tissues consistent with patterns seen for expression of $P$. tremula $\times$ P. tremuloides PtUGDH and A. thaliana AtUGDH genes. PtUGDH expression occurs predominantly in developing xylem and young leaves, with some expression in mature and apical leaves (Johansson et al. 2002), and AtUGDH is mainly expressed in young growing tissues and is not expressed in mature tissues (Seitz et al. 2000).

The regulation of gene expression is a complex process controlled by multiple factors that can act during transcription, RNA splicing, and mRNA translation

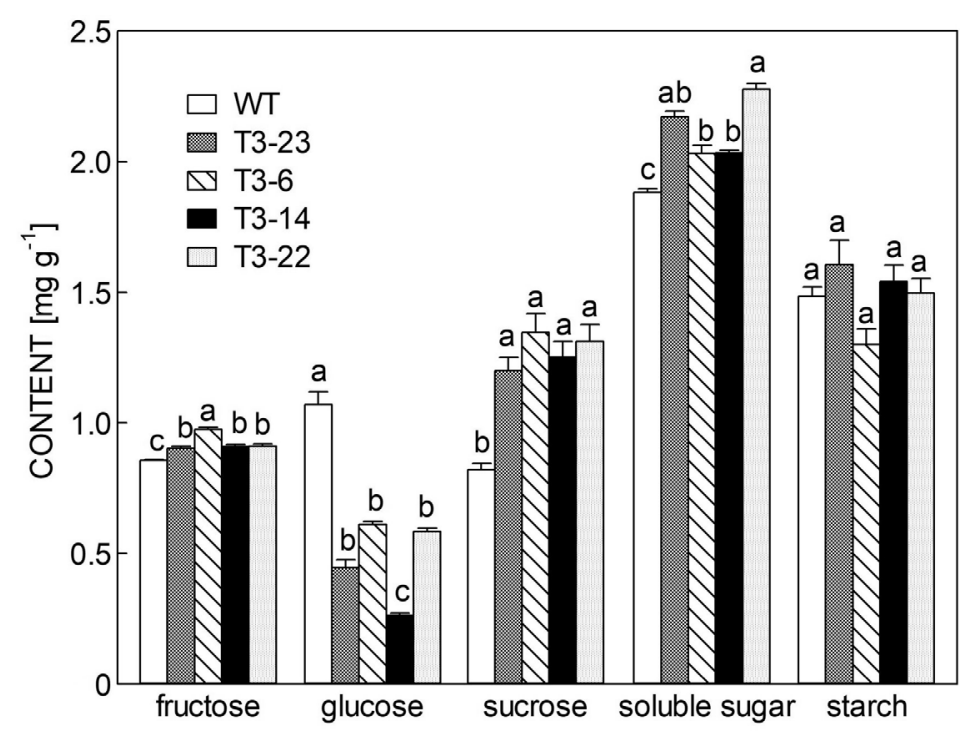

Fig. 5. Sucrose, glucose, fructose, soluble sugar, and starch content in fresh mass in the fifth rosette leaf of 30-d-old wild-type and transgenic Arabidopsis thaliana plants. Error bars represent SDs of three independent biological replicates. Different letters indicate significant differences at $P<0.05$.

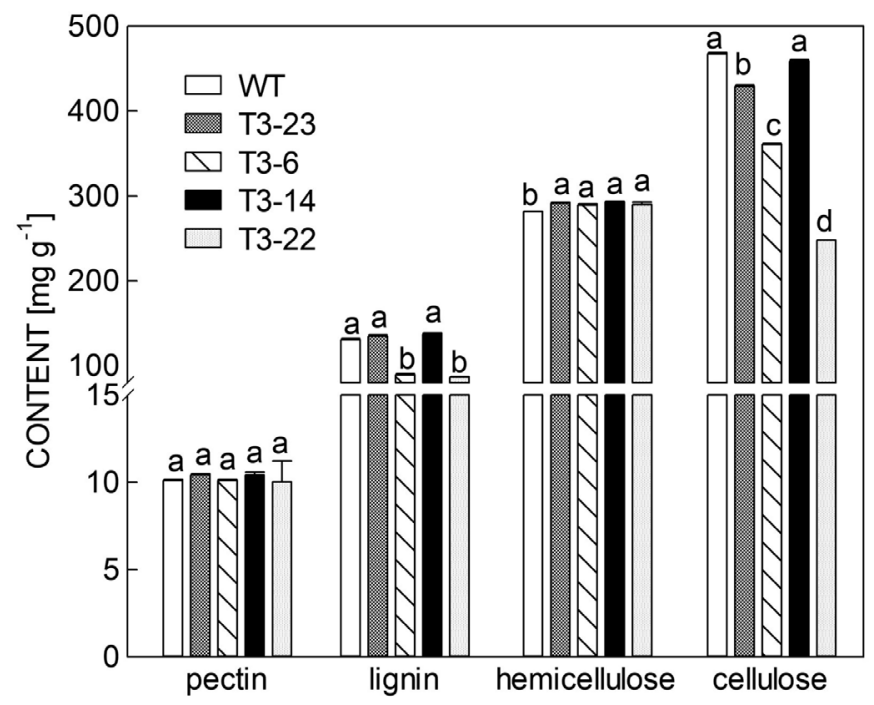

Fig. 6. Lignin, cellulose, and hemicellulose content in dry mass, and pectin content in fresh mass in stems of 45-d-old wild-type and transgenic Arabidopsis thaliana plants. Error bars represent SDs of three independent biological replicates. Different letters indicate significant differences at $P<0.05$. 
(Mithra et al. 2017). Regulation of eukaryotic gene expression is generally divided into multiple levels, with the transcription level being the most critical. Cis-regulatory elements located in the promoter region play important roles in transcriptional regulation, and therefore functional analysis of the upstream promoter is important for understanding the transcriptional regulation mechanism of genes. In this study, we cloned the $B p U G D H$ gene promoter, and sequence analysis showed that some cis-elements involved in responses to low temperature and phytohormones, including $\mathrm{GA}_{3}$, IAA, ETH, and MeJA, were present in the $B p U G D H$ promoter, suggesting that $B p U G D H$ is involved in the plant response to cold and in regulation by phytohormones. GUS staining showed that, with GUS expression driven by the $B p U G D H$ promoter, GUS was strongly expressed in young and developing tissues of transgenic $A$. thaliana plants, and its expression was strongly induced by low temperature, $\mathrm{GA}_{3}$, IAA, MeJA, and ETH (Fig. 2) consistent with the function of
UGDH and the cis-elements present in the $B p U G D H$ promoter.

Multiple $U G D H$ isoforms were indentified in plant genome, such as in Arabidopsis, the $U G D H$ gene family is represented by four highly similar $U G D H$ isoforms and five $U G D H$ pseudogenes (Seitz et al. 2000, Reiter and Vanzin 2001, Klinghammer and Tenhaken 2007). Moreover, in poplar, at least two isoforms were reported (Johansson et al. 2002). In the L. gmelinii genome, two copies of $U G D H$ exist (Li et al. 2017). In Eucalyptus more than one copy of $U G D H$ is present (Labate et al. 2010). In suspension-cultured maize cells, several lines of evidence indicated the existence of three isoforms with widely differing substrate affinities (Kärkönen et al. 2005). In culm tissue of Saccharum spp., another gramineous plant, only one UGDHwas detected (Turner and Botha 2002). In our previous study, five UGDH transcripts were found in the transcriptome database of paper mulberry (unpublished data).

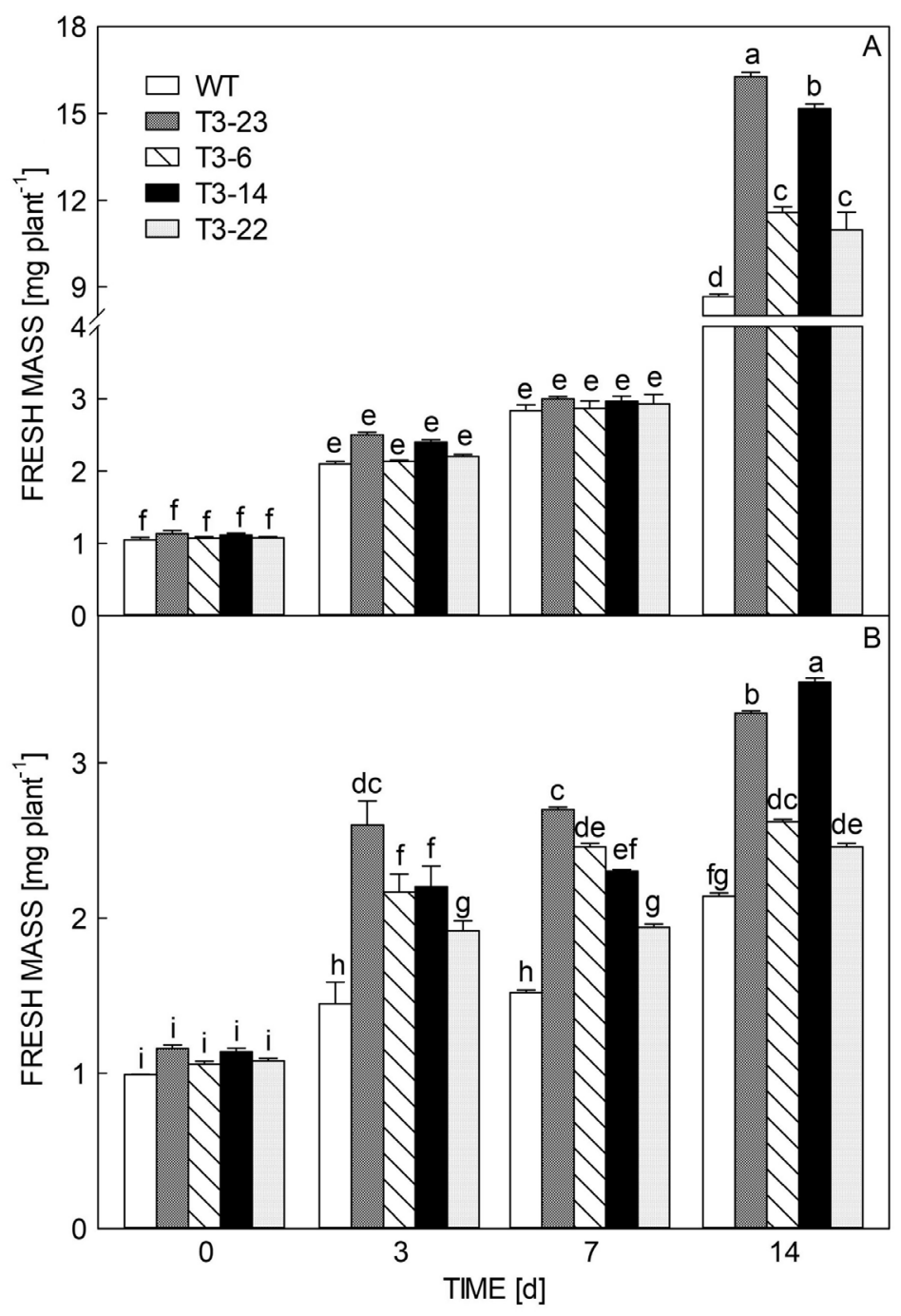

Fig. 7. Cold-tolerance of Broussonetia papyifera uridine diphosphate glucose dehydrogenase transgenic plants. Wild-type and four transgenic lines first cultured on a half strength Murasige and Skoog medium for $8 \mathrm{~d}$ were subsequently cultured at $22^{\circ} \mathrm{C}(A)$ or $4{ }^{\circ} \mathrm{C}$ $(B)$ for $0,3,7$, and $14 \mathrm{~d}$, and fresh masses were measured. Error bars represent SDs of three independent biological replicates. Different letters indicate significant differences at $P<0.05$. 
Each $U G D H$ isoform exhibits a different expression pattern during plant development. In Ipomoea batatas, $I b U D P G H 1$ is strongly expressed in the tuberous roots, IbUDPGH2 and IbUDPGH5 are expressed in stems, and the other $I b U G D H$ genes are slightly expressed in different tissues (Lai et al. 2014). Moreover, four functional $U G D H$ genes and one pseudogene of Arabidopsis exhibit distinct tissue-specific expression patterns (Klinghammer and Tenhaken 2007). During germination and the vegetative phase, the expression patterns of $A t U G D H 2,3$, and 4 were very similar, with strong expression in primary roots, root tips, young root hairs, and calyptras, while AtUGDH1 showed an almost inverse organ-specific pattern with low expression in cotyledons. During the reproductive phase, all $A t U G D H$ activities were detected in stigma, filaments, and mature pollen, while each $A t U G D H$ gene showed a different expression pattern in sepals, petals, pollen sacks, and siliques. In this work, GUS staining showed that the $B p U G D H$ promoter was active in all organs of transgenic A. thaliana during the growth and development phases, with a higher activity in young leaves, roots, petals, calyx, stamens, and siliques, indicating the importance of $B p U G D H$ regulation in the growth and development of paper mulberry. No BpUGDH::GUS activity was detected in seeds of transgenic A. thaliana, suggesting the possibility that the $B p U G D H$ promoter could be used to drive functional genes for genetic improvement of grain crops.

Overexpression of $B p U G D H$ enhanced the vegetative growth of transgenic $A$. thaliana plants, similar to results of our previous study of L. gmelinii $U G D H(\mathrm{Li}$ et al. 2017). Overexpression of $L g U G D H$ significantly enhances vegetative growth in transgenic $A$. thaliana (Nossen ecotype) during all growth stages (Li et al. 2017). In this study, no obvious difference between wild-type and transgenic plants overexpressing $B p U G D H$ was found during the early stage, while a different growth phenotype was observed after transplanting to soil (Fig. 4B,C). The difference in growth phenotype was due to the different ecotype backgrounds of the plants; the $A$. thaliana Nossen ecotype was used for overexpression of L. gmelinii UGDH (Li et al. 2017), while the Columbia ecotype was used for overexpression of $B p U G D H$ in this study.

The Z. mays knockout mutant ugdh-Al reduces pentosan biosynthesis, suggesting that $U G D H$ is a critical factor in sugar metabolism (Kärkönen and Fry 2006). Our results showed that the contents of sucrose, fructose, and soluble sugars in leaves of $B p U G D H$ transgenic $A$. thaliana were significantly higher than those of wildtype plants (Fig. 5), indicating that $B p U G D H$ may modify carbon allocation in favour of soluble sugars. However, the glucose content was significantly reduced in the transgenic plants, while no significant difference in starch content was observed. Glucose is converted by UGPase to UDP-Glc. UDP-Glc can be converted by UGDH to UDP-GlcA, which is involved in hemicellulose and pectin synthesis. Therefore, the reduction in glucose content may be caused by substrate consumption in hemicellulose and pectin biosynthetic pathways. Our results indicated that the accumulated sugar was probably not stored in leaves in the form of starch, but formed a pool of available soluble sugars to accelerate metabolism and enhance vegetative growth.

Plant cell walls play a crucial role in plant growth, defense, and structural integrity, and contain many polysaccharides including cellulose, hemicelluloses, and tannins. Hemicellulose is an important factor for secondary cell wall biosynthesis and accounts for half of the primary cell wall biomass (Delmer et al. 1988, Witt 1992, Gibeaut 2000, Seifert 2004, Kärkönen and Fry 2006, Sato et al. 2013). The hemicellulose content of transgenic plants overexpressing $B p U G D H$ increased significantly compared to the wild-type plants (Fig. 6), indicating that $B p U G D H$ plays an important role in glucose metabolism, especially in hemicellulose biosynthesis. This result is similar to those of our previous research on L. gmelinii $U G D H$ (Li et al. 2017). The cellulose content decreased significantly, especially in the transgenic lines T3-23, T3-6 and T3-22. The reason may be that the overexpression of $B p U G D H$ significantly increased the activity of UGDH in transgenic plants. As a result, a large amount of UDP-Glc is consumed, and cellulose which is a main component of the cell wall is formed by the binding of dextran, and thus overexpression of $B p U G D H$ causes a decrease in the cellulose content in the stem of the transgenic $A$. thaliana. Control of UGDH activity by feedback inhibition seems to be a common mechanism controlling the enzymes involved in sugar-nucleotide interconversions. The control for this system might occur at the transcriptional level of genes encoding kinetically distinct enzymes (Seifert 2004). In our experiments, the transgenic line T3-14 with the highest UGDH activity had sugar content lower than other transgenic plants. It seems that, in transgenic $A$. thaliana, nucleoside sugars at low concentrations function as a feedback stimulator, activating the synthesis of UDPGlcA and thus potentially its own synthesis (Kärkönen and Fry 2006). The increase in the hemicellulose and soluble sugar content indicated that $B p U G D H$ overexpression may contribute to plant adaptation to stress, which was confirmed by the cold tolerance exhibited by the transgenic plants in this study. Previous research has shown that transgenic plants overexpressing L. gmelinii UGDH showed stronger cold tolerance ( $\mathrm{Li}$ et al. 2017). Our results strongly suggest that $B p U G D H$ may be a good candidate gene for improving cold resistance, accelerating fiber cell development, and enhancing plant vegetative growth.

\section{References}

Aubertin, C.: Paper mulberry (Broussonetia papyrifera) in Lao PDR: a successful example of forest product domestication. In: Kusters, K., Belcher, B.: Forest Products, Livelihoods and Conservation: Case-studies of Non-timber Forest Product Systems. Vol. 1. Pp. 227-246. CIFOR, Bogor 2004.

Campbell, R.E., Mosimann, S.C., Rijn, I.V.D., Tanner, M.E., Strynadka, N.C.: The first structure of UDP-glucose dehydrogenase reveals the catalytic residues necessary for the two-fold oxidation. - Biochemistry 39: 7012-7023, 2000.

Delmer, D.P., Stone, B.A., Preiss, J.: Biosynthesis of plant cell walls. - Biochem. Plants 14: 373-420, 1988. 
Gibeaut, D.M.: Nucleotide sugars and glycosyltransferases for synthesis of cell wall matrix polysaccharides. - Plant Physiol. Biochem. 38: 69-80, 2000.

Gibeaut, D.M., Carpita, N.C.: Biosynthesis of plant cell wall polysaccharides. - FASEB J. 8: 904-915, 1994.

Griffith, C.L., Klutts, J.S., Zhang, L.J., Levery, S.B., Doering, T.L.: UDP-glucose dehydrogenase plays multiple roles in the biology of the pathogenic fungus Cryptococcus neoformans. - J. biol. Chem. 279: 51669-51676, 2004.

He, Q.H., Qiao, D.R., Zhang, Q.L., He, S.J., Li, Y., Bai, L.H., Yang, Z.R., Cao, Y.: Cloning and expression studies of the Dunaliella salina UDP-glucose dehydrogenase cDNA. DNA Sequence 16: 202-206, 2005.

Johansson, H., Sterky, F., Amini, B., Lundeberg, J., Kleczkowski, L.A.: Molecular cloning and characterization of a cDNA encoding poplar UDP-glucose dehydrogenase, a key gene of hemicellulose/pectin formation. - Biochim. Biophys. Acta. 1576: 53-58, 2002.

Kärkönen, A., Fry, S.C.: Novel characteristics of UDP-glucose dehydrogenase activities in maize: non-involvement of alcohol dehydrogenases in cell wall polysaccharide biosynthesis. Planta 223: 858-870, 2006.

Kärkönen, A., Murigneux, A., Martinant, J., Pepey, E., Tatout, C., Dudley, B., Fry, S.: UDP-glucose dehydrogenases of maize: a role in cell wall pentose biosynthesis. - Biochem. J. 391: 409-415, 2005.

Kleczkowski, L.A., Geisler, M., Ciereszko, I., Johansson, H.: UDP-glucose pyrophosphorylase. An old protein with new tricks. - Plant Physiol. 134: 912-918, 2004.

Kim, G.T., Tsukaya, H., Saito, Y., Uchimiya, H.: Changes in the shapes of leaves and flowers upon overexpression of cytochrome P450 in Arabidopsis. - Proc. nat. Acad. Sci. USA 96: 9433-9437, 1999.

Klinghammer, M., Tenhaken, R.: Genome-wide analysis of the UDP-glucose dehydrogenase gene family in Arabidopsis, a key enzyme for matrix polysaccharides in cell walls. - J. exp. Bot. 58: 3609-3021, 2007.

Kuang, Y., Xi, D., Li, J., Zhu, X., Zhang, L.: Traffic pollution influences leaf biochemistries of Broussonetia papyrifera. Open J. Forestry 2: 71-76, 2012.

Labate, M.T.V., Bertolo, A.L.F., Nascimento, D.D.D., Gutmanis, G., Andrade, A.D., Rodrigues, M.J.C., Camargo, E.L.O., Boaretto, L.F., Moon, D.H., Bragatto, J., Labate, C.A.: Cloning and endogenous expression of a Eucalyptus grandis UDP-glucose dehydrogenase cDNA. - Gene. mol. Biol. 33: 686-695, 2010.

Lai, X.J., Gu, Y.H., Tao, X., Zhang, Y.Z., Wang, H.Y.: Cloning and characterization of uridine diphosphate glucose dehydrogenase gene from Ipomoea batatas. - Russ. J. Plant Physiol. 61: 298-308, 2014

Lescot, M., Déhais, P., Thijs, G., Marchal, K., Moreau, Y., Peer Y.V.D., Rouzé, P., Rombauts, S.: Plant CARE, a database of plant cis-acting regulatory elements and a portal to tools for in silico analysis of promoter sequences. - Nucl. Acids Res. 30: 325-327, 2002.

Li, G.Y., Hu, N., Ding, D.X., Zheng, J.F., Liu, Y.L., Wang, Y.D., Nie, X.Q.: Screening of plant species for phytoremediation of uranium, thorium, barium, nickel, strontium and lead contaminated soils from a uranium mill tailings repository in South China. - Bull. environ. Contam. Toxicol. 86: 646-652, 2011.

Li, M.R., Li, H.Q., Jiang, H.W., Wu, G.J.: Establishment of a highly efficient Agrobacterium tumefaciens-mediated leaf disc transformation method for Broussonetia papyrifera. Plant Cell Tissue Organ Cult. 93: 249-255, 2008.

Li, M.R., Li, Y., Li, H.Q., Wu, G.J.: Overexpression of AtNHX5 improves tolerance to both salt and drought stress in Broussonetia papyrifera (L.) Vent. - Tree Physiol. 31: 349357, 2011.

Li, N.N., Chen, L., Li, X.H., Li, Q., Lin, X.F., Zhang, W.B., Takechi, K., Takano, H.: Overexpression of UDP-glucose dehydrogenase from Larix gmelinii enhances growth and cold tolerance in transgenic Arabidopsis thaliana. - Biol. Plant. 61: 95-105, 2017.

Li, N.N., Wang, L., Zhang, W.B., Takechi, K., Takano, H., Lin, X.F.: Overexpression of UDP-glucose pyrophosphorylase from Larix gmelinii enhances vegetative growth in transgenic Arabidopsis thaliana. - Plant Cell Rep. 33: 779-791, 2014.

Li, Y., Li, G.: [Cloning and sequence analysis of actin gene from Broussonetia papyrifera.] - Biotechnology 17: 1-3, 2007. [In Port.]

Lin, X.F., Minamisawa, N., Takechi, K., Zhang, W.B., Sato, H., Takio, S., Tsukaya, H., Takano, H.: Isolation and characterization of the Larix gmelinii ANGUSTIFOLIA ( $L g A N)$ gene. - Planta 228: 601-608, 2008.

Liu, F., Huang, Y., Guo, Q.Q., Zhang, X.W., Li, L.Y., Deng, J., Xie, L.L.: [Cloning and expression of UDP-glucose dehydrogenase (UDPGDH) cDNA in ramie (Boehmeria nivea (Linn.) Gaud).] - Sci. agr. sin. 41: 3542-3548, 2008. [In Chin.]

Livak, K.J., Schmittgen, T.D.: Analysis of relative gene expression data using realtime quantitative PCR and the $2^{-\Delta \Delta C t}$ method. - Methods 25: 402-408, 2001.

Matsumoto, T., Tanaka, T., Sakai, H., Amano, N., Kanamori, H., Kurita, K., Kikuta, A., Kamiya, K., Yamamoto, M., Ikawa, H., Fujii, N., Hor, K., Itoh, T., Sato, K.: Comprehensive sequence analysis of 24783 barley full-length cDNAs derived from 12 clone libraries. - Plant Physiol. 156: 20-28, 2011.

Mithra, S.V.A., Kulkarni, K., Srinivasan, R.: Plant promoters: characterization and applications in transgenic technology. In: Abdin, M.Z., Kiran, U., Kamaluddin, A.A. (ed.): Plant Biotechnology: Principles and Applications. Pp. 117-172. Springer, Singapore 2017.

Nagpal, U.M., Bankar, A.V., Pawar, N.J., Kapadnis, B.P., Zinjarde, S.S.: Equilibrium and kinetic studies on biosorption of heavy metals by leaf powder of paper mulberry (Broussonetia papyrifera). - Water Air Soil Pollut. 215: 177-188, 2011.

Pang, C.Y., Wang, H., Pang, Y., Xu, C., Jiao, Y., Qin, Y.M., Western, T.L., Yu, S.X., Zhu, Y.X.: Comparative proteomics indicates that biosynthesis of pectic precursors is important for cotton fiber and Arabidopsis root hair elongation. - Mol. cell. Proteomics 9: 2019-2033, 2010.

Peng, X.J., Teng, L.H., Yan, X.Q., Zhao, M.L., Shen, S.H.: The cold responsive mechanism of the paper mulberry: decreased photosynthesis capacity and increased starch accumulation. BMC Genomics 16: 898, 2015.

Reiter, W.D., Vanzin, G.F.: Molecular genetics of nucleotide sugar interconversion pathways in plants. - Plant mol. Biol. 47: 95-113, 2001.

Rigg, G.P., Barrett, B., Roberts, I.S.: The localization of $\mathrm{KpsC}$, S and $\mathrm{T}$, and KfiA, C and D proteins involved in the biosynthesis of the Escherichia coli K5 capsular polysaccharide: evidence for a membrane-bound complex. - Microbiology 144: 2905 2914, 1998.

Robertson, D., Smith, C., Bolwell, G.P.: Inducible UDP-glucose dehydrogenase from French bean (Phaseolus vulgaris L.) locates to vascular tissue and has alcohol dehydrogenase activity. - Biochem. J. 313: 311-317, 1996.

Sato, K., Hihara, E., Yamaguchi, M., Kanahama, K., Kanayama, Y.: Possible role of a peach homolog of UDP-glucose dehydrogenase in fruit development. - Scientia hort. 150: 195200, 2013.

Seifert, G.J.: Nucleotide sugar interconversions and cell wall 
biosynthesis: how to bring the inside to the outside. - Curr. Opin. Plant Biol. 7: 277-284, 2004.

Seitz, B., Klos, C., Wurm, M., Tenhaken, R.: Matrix polysaccharide precursors in Arabidopsis cell walls are synthesized by alternate pathways with organ-specific expression patterns. - Plant J. 21: 537-546, 2000.

Stewart, D.C., Copeland, L.: Uridine 5'-diphosphate-glucose dehydrogenase from soybean nodules. - Plant Physiol. 116: 349-355, 1998.

Strominger, J.L., Kalckar, H.M., Axelrod, J., Maxwell, E.S.: Enzymatic oxidation of uridine diphosphate glucose to uridine disphosphate glucuronic acid. - J. amer. chem. Soc. 76: 6411-6412, 1954

Sun, J., Liu, S.F., Zhang, C.S., Yu, L.N., Bi, J., Zhu, F., Yang, Q.L.: Chemical composition and antioxidant activities of Broussonetia papyrifera fruits. - PLoS ONE 7: e32021, 2012.

Sun, J.W., Peng, X.J., Fan, W.H., Tang, M.J., Liu, J., Shen, S.H.: Functional analysis of BpDREB2 gene involved in salt and drought response from a woody plant Broussonetia papyrifera. - Gene 535: 140-149, 2014.
Tenhaken, R., Thulke, O.: Cloning of an enzyme that synthesizes a key nucleotide sugar precursor of hemicellulose biosynthesis from soybean: UDP-glucose dehydrogenase. - Plant Physiol. 112: 1127-1134, 1996.

Turner, W., Botha, F.C.: Purification and kinetic properties of UDP-glucose dehydrogenase from sugarcane. - Arch. Biochem. Biophys. 407: 209-216, 2002.

Wang, J., Li, A., Wang, C.G., Song, W.Q., Chen, C.B.: [Cloning and expression of UDP-glucose dehydrogenase cDNA in larix kaempferi.] - Forest Res. 26: 76-81, 2013. [In Chin.]

Winter, H., Huber, S.C.: Regulation of sucrose metabolism in higher plants: localization and regulation of activity of key enzymes. - Crit. Rev. Plant Sci. 19: 253-289, 2000.

Witt, H.J.: UDP-glucose metabolism during differentiation and dedifferentiation of Riella helicophylla. - J. Plant Physiol. 140: 276-281, 1992.

Yan, J., Wu, P., Du, H., Zhang, Q.: First report of black spot caused by Colletotrichum gloeosporioides on paper mulberry in China. - Plant Dis. 95: 880-880, 2011. 